\title{
La enseñanza de lenguas extranjeras y la Teoría de las Inteligencias Múl- tiples de Gardner. Reflexiones sobre la importancia de la emoción en el aprendizaje de lenguas extranjeras
}

\author{
Ángel Gabarrón Pérez* \\ Universidad Nacional de Educación a Distancia (España)
}

\author{
PALABRAS CLAVE \\ Enseñanza de lenguas \\ extranjeras \\ Inteligencias múltiples \\ Inteligencia emocional \\ Profesor emocional
}

\begin{abstract}
RESUMEN
En el presente artículo se recoge una reflexión sobre la influencia de la Teoría de las Inteligencias Múltiples de Howard Gardner en la enseñanza de lenguas extranjeras a través de la revisión de literatura tradicional y contemporánea sobre el tema, estableciendo una retrospección que parte del "Nivel Umbral" (documento elaborado por expertos para el Consejo de Europa, que habría de ser la antesala del Marco Común Europeo de Referencia para las Lenguas (MCERL)), para continuar con las diferentes teorías pedagógicas que han influido en la enseñanza de lenguas durante las décadas de los 70 y 80, hasta llegar a la Teoría de las Inteligencias Múltiples.. En consecuencia, reflexionaremos sobre la forma en que cada individuo desarrolla en mayor o menor medida cada una de las inteligencias consideradas en la teoría de Gardner durante el proceso de enseñanza-aprendizaje de las lenguas extranjeras. Finalmente, teniendo en cuenta la importancia de la inteligencia emocional en el aprendizaje de una lengua, analizaremos el concepto del docente de lenguas como un "profesor emocional", un gestor de sus propias emociones y de las emociones del discente, capaz de generar el escenario válido para el desarrollo de un proceso adecuado de enseñanza-aprendizaje de una lengua extranjera, y la importancia de las TIC en dicho proceso.
\end{abstract}

Foreign language teaching and Gardner's Theory of Multiple Intelligences. Afterthoughts on the importance of emotions in foreign language learning

\section{ABSTRACT}

This article is an afterthought, on the influence of Howard Gardner's Theory of Multiple Intelligences in foreign language teaching, establishing a retrospective that starts from the "Threshold Level", a document prepared by experts for the Council of Europe, which would be the prelude to the Common European Framework of Reference for Languages, to continue with the different pedagogical theories that have influenced the teaching of non-maternal languages during the 70s and 80s, and ends with the Theory of Multiple Intelligences. Therefore, we will think over the teaching and learning process of foreign languages, establishing that every individual develops each intelligence considered in

Universidad Nacional de Educación a Distancia

Autor de correspondencia: * Ángel Gabarrón Pérez. Email: agabarronperez@gmail.com. Dirección postal: Avenida Teniente General Muslera, n. ${ }^{\circ}$ 1, B. A, 4. ${ }^{\circ}$ 1, Ceuta (CP 51002). Teléfono: 661027953

Recibido: 02/07/2019 - Aceptado: 13/12/2019

Revista de Formación del Profesorado e Investigación Educativa

Facultad de Formación del Profesorado y Educación

Universidad de Oviedo - Universidá d'Uviéu - University of Oviedo

Julio - Diciembre 2019

ISSN: $2340-4728$ 
this theory to a greater or lesser extent. Finally, and taking into account the importance of emotional intelligence in the learning of a non-native language, we will analyze the concept of the language teacher as an "emotional teacher", a manager of his own emotions and the learner's, capable of generating the valid scenario for the development of an adequate teaching-learning process of a foreign language, and how important ICT are for the implementation of this process.

\section{Introducción}

En el año 1971 se celebró en Rüschlikon (Suiza) un Simposio sobre La Enseñanza de Lenguas Modernas en la Educación de los Adultos, como respuesta al flujo migratorio de trabajadores entre países de la Comunidad Económica Europea (CEE). Ante esta situación, el Comité de Educación Extraescolar y Desarrollo Cultural del Consejo de Europa encargó a un equipo de expertos, dirigidos por el Director del Centro de Información e Investigaciones para la Enseñanza de Lenguas (CILT) de Londres, John Leslie Melvin Trim, un documento que determinara el "Nivel Umbral" (The Threshold Level) o el grado mínimo de dominio que debía alcanzar el aprendiz de una lengua no materna para poder ser usada en situaciones de la vida cotidiana y en contextos reales y habituales (Slagter \& Van Ek, 1979). El resultado fue "The Threshold Level in a European Unit/Credit System for Modern Language Learning by Adults" publicado por J.A. Van Ek, con introducción de J.L.M. Trim y apéndice de L.G. Alexander (1975). En dicho documento se determinan los principios fundamentales en los que debe basarse la enseñanza de lenguas modernas, partiendo de la enseñanza del inglés, y estableciendo como eje central el uso de la lengua por parte de los hablantes más que la lengua en sí. Este documento finalmente se convertiría en la antesala de lo que posteriormente sería el Marco Común Europeo de Referencia para las Lenguas (MCER) (Trim, 2007).

Desde este momento la enseñanza de lenguas extranjeras será considerada un proceso instructivo centrado en los discentes, que deberán participar activamente en su proceso de aprendizaje para poder tomar decisiones en la lengua meta de forma autónoma, tratando contenidos que guarden relación con la realidad y el mundo que les rodea (Salinas et al., 2012). Para ello el enfoque comunicativo ocupará un lugar relevante, y los docentes de lenguas extranjeras deberán implementar una metodología activa, basada en proyectos a realizar en la lengua meta (Van Ek \& Alexander, 1980, p. 12).

La enseñanza de lenguas extranjeras quedó determinada por el Nivel Umbral del Consejo de Europa durante la década de los 80. Se establece un tipo de aprendizaje de lenguas no maternas basado fundamentalmente en el enfoque comunicativo: los docentes crean escenarios de aprendizaje donde se simulan situaciones de comunicación reales o verosímiles, el texto real y contextualizado es la unidad de comunicación, se implementa un trabajo con los aprendientes por parejas o grupos y se establece como objetivo el desarrollo gradual de las cuatro destrezas o habilidades en una lengua (lectura, escritura, audición y habla) (Moreno, 2009; Ramírez \& Acosta, 2015).

Desde ese momento y en los años posteriores se desarrollará una serie de teorías que añadirán elementos innovadores a la enseñanza de lenguas extranjeras. Una de ellas es la Teoría del Input Comprensible de Stephen Krashen (1985), quien establece, por una parte, que el aprendizaje de una lengua no materna resulta de la combinación de la teoría de la Zona de Desarrollo Próximo de Lev Vigotsky (1939) y la teoría de Noam Chomsky (1986) sobre el lenguaje en cuanto objeto natural que forma parte de la dotación biológica de los hablantes y sostiene, por otra parte, que el conocimiento de una lengua aumenta en el aprendiz cuando este recibe información que puede comprender y que está situada a un nivel ligeramente superior a su capacidad (Morales \& Barrientos, 2016; Schütz, 2007).

Tomando la Teoría del Input Comprensible como punto de partida, Tracy Terrell, profesora de español como lengua no materna, y Stephen Krashen desarrollan el Enfoque Natural (1983), que tendrá una gran importancia en las metodologías de enseñanza de idiomas en Europa y Estados Unidos (Kiraly \& Hernández, 2019; Lizasoain \& Mansilla, 2018). Esta teoría postula que el docente de lenguas extranjeras deberá crear actividades que despierten una variedad de estímulos e intereses de tal forma que aporten un input comprensible a los discentes (Terrell, 1986). Krashen y Terrell señalan tres estadios en el proceso de aprendizaje de una lengua no materna: el Estadio de la Pre-producción o período de silencio, donde se produce el desarrollo de la comprensión auditiva; el Estadio de la Producción Precoz, donde el error, que no debe ser corregido salvo en casos de gravedad, adquiere una gran importancia para el desarrollo del proceso de aprendizaje, ya que la importancia del significado prevalece sobre la forma; y la Producción Discursiva, donde el aprendiz realizará actividades de interacción y diálogo como la asunción de roles, el debate y el trabajo en equipo (Krashen \& Terrell, 1983).

A principios de los 90 surge una nueva propuesta, cuyos orígenes se encuentran en el mundo anglosajón. Los enfoques comunicativos evolucionaron hacia el llamado Enfoque por Tareas (Lee, 2016; Zanón, 1990), que es un programa de aprendizaje cuyas actividades están focalizadas en el uso de la lengua, alejándose de la sintaxis o de aquellas actividades basadas en enfoque nocionales o funcionales (Vez, 2018). Su objetivo principal es promover el aprendizaje mediante actividades que fomenten el uso de la lengua en situaciones reales, incluyendo de esta forma los procesos de comunicación en el aprendizaje del idioma (Zanón, 1995).

Son muchos los autores que han ofrecido una definición de tarea (Breen, 1987; Long, 1985; Menéndez, 2007; Nunan, 1989; Puren, 2012). Las primeras definiciones se refieren a ella como aquella actividad realizada en el aula que intenta simular situaciones que se producen fuera de ella (Candlin, 1990). Pero las definiciones posteriores introdujeron otras variantes: está provista de una adecuada estructura pedagógica, permite las aportaciones personales y la intervención activa de los discentes, centra la atención del aprendiz en el contenido de sus mensajes y facilita en algunos momentos de su ejecución prestar atención a la forma lingüística (Mass et al., 2010). Es necesario distinguir entre tareas finales, que son aquellas enfocadas desde una visión global de la propia tarea, y las tareas posibilitadoras, con las que se aluden a los pasos previos e intermedios que son esenciales para que el aprendiz evolucione en el desarrollo de sus capacidades hasta llegar a la consecución de la propia tarea final (Estaire, 1990; Rendine, 2018).

Como consecuencia de todas estas nuevas tendencias pedagógicas aplicadas a la enseñanza de lenguas extranjeras, surge el Aprendizaje Cooperativo (Fontes et al., 2019) como una propuesta metodológica en la que el discente se sitúa en el centro del proceso de aprendizaje, y la característica principal es la distribución del aula en diferentes equipos y grupos de trabajo (Slavin, 1980). Generalmente, todos los modelos de Aprendizaje Cooperativo tienen una serie de características comunes: un proceso positivo de interdependencia entre los miembros que conforman el grupo de trabajo; una interacción grupal directa; un impulso y una motivación por asumir responsabilidades individualmente y en grupo 
por parte de los discentes; el desarrollo de habilidades sociales y la implementación de un proceso reflexivo sobre el aprendizaje (Lie, 2002). Aplicado a la enseñanza de lenguas extranjeras, la variable emocional ha sido la principal aportación del Aprendizaje Cooperativo (Godoy \& Madinabeitia, 2016). El aprendizaje se desarrolla en un contexto donde se produce una disminución de la presión y la ansiedad, por lo que la motivación aumenta proporcionalmente, desarrollando en el aprendiz una predisposición afectiva hacia el aprendizaje de la lengua, elevando su autoestima (Trujillo, 2002; Valladares, 2016).

\section{La Teoría de las Inteligencias Múltiples y las emociones en la enseñanza de lenguas extranjeras}

La Teoría de las Inteligencias Múltiples de Howard Gardner (1993) introdujo una concepción multidimensional de los procesos de aprendizaje, ya que dicha teoría postulaba que todos los individuos poseen diferentes tipos de inteligencias desarrolladas de manera diferente, que pueden ser potenciadas mediante la práctica y el entrenamiento educativo (Suárez et al., 2010). La teoría de Gardner está estrechamente relacionada con las metodologías innovadoras en la enseñanza de lenguas extranjeras, especialmente todas aquellas que guardan relación con el Aprendizaje Integrado de Contenidos en Lenguas Extranjeras, o AICLE (en inglés Content and Language Integrated Learning, o su acrónimo CLIL) (Carrillo \& López, 2014; Griva \& Deligianni, 2017). Por otra parte, determinadas actitudes del discente durante el proceso de aprendizaje de una lengua no materna, como la inhibición, la autoestima o la motivación, están muy vinculados con la aplicación de la Teoría de las Inteligencias Múltiples y su fundamentación desde un punto de vista psicopedagógico (Diaz et al., 2017)

Así pues, en el aprendiente coinciden ocho tipos de inteligencias: la inteligencia lingüística, o la habilidad para el uso creativo y especial de la lengua; la inteligencia lógica y matemática, o la habilidad para desarrollar un pensamiento racional; la inteligencia espacial, o la habilidad para la formación de imágenes y modelos del mundo que nos rodea; la inteligencia musical, o la habilidad para mostrar sensibilidad por la música, así como para su composición; la inteligencia corporal y kinestética, o la capacidad de desarrollar una buena coordinación corporal; la inteligencia interpersonal, o la habilidad para la socialización con otros individuos; la inteligencia intrapersonal, o el óptimo conocimiento por parte del individuo de su propio cuerpo; $y$ la inteligencia naturalista, o la habilidad para entender y desenvolverse en el medio natural (Gardner \& Hatch, 1989).

De todas ellas, obviamente la inteligencia lingüística debe tenerse muy en cuenta en los procesos de enseñanza y aprendizaje de lenguas extranjeras. De hecho, el propio Gardner creó el término de "inteligencia lingüística" (Mercadé, 2016; Yepes et al., 2017), aunque reconocía que en el aprendizaje de un idioma confluyen otros factores a tener en cuenta además de los lingüísticos, llegando a la conclusión de que todas las inteligencias influyen de alguna manera en el proceso de adquisición de una lengua no materna (Acevedo, 2018), y la intervención de los sentidos situaban a dicho proceso en un plano multisensorial (Carrillo \& López, 2014). La Teoría de las Inteligencias Múltiples demostró que la lengua no debe ser estudiada solamente desde el plano gramatical, o al menos éste no debe priorizarse sobre otros planos que incluyen otros tipos de inteligencias (Valdivia et al., 2019). Del mismo modo, la teoría de Gardner determinó lo trascendental de la implicación de los sentimientos y las emociones en el proceso de aprendizaje, en general, y en el de lenguas extranjeras en particular (López, 2016).

Pero debemos ir más allá de las inteligencias interpersonal e intrapersonal para llegar a la conclusión de que el aprendizaje de lenguas extranjeras es un proceso en el que intervienen en mayor o menor grado las otras inteligencias descritas en la teoría de Gardner (García, 2018). De este modo, además de vincular la reflexión y el razonamiento con la inteligencia intrapersonal y la necesidad de comunicarnos con la inteligencia interpersonal, podemos establecer una relación entre los diferentes movimientos que los hablantes realizan con los diferentes órganos del cuerpo vinculados al habla para producir los fonemas correspondientes a cada lengua con la inteligencia cinético-corporal, entre el uso lógico de las estructuras gramaticales de una lengua y la inteligencia lógico-matemática o entre la musicalidad de la entonación de un idioma y la inteligencia musical (Acevedo, 2018)

Y llegamos de esta forma al concepto de inteligencia emocional, cuyo origen se encuentra en dos profesores de psicología de la universidad de Yale, Peter Salovey y John D. Mayer (1990), aunque fue el trabajo de difusión del escritor y periodista Daniel Goleman (1996) el que permitió que dicho concepto fuese conocido mundialmente, refiriéndose a la inteligencia emocional como la habilidad y capacidad que desarrollan los individuos para gestionar sus emociones y para empatizar con las emociones de los demás (Mayer \& Salovey, 1997). El proceso de desarrollo de la inteligencia emocional comienza con el conocimiento de nuestras propias emociones o fase de autoconciencia, la gestión de dichas emociones o fase de autorregulación, el uso de las emociones para conseguir la motivación, por ejemplo, en el proceso de aprendizaje, o fase de automotivación, la capacidad de reconocer las emociones de otros individuos o fase de empatía, y finalmente, la gestión de las relaciones humanas o fase de socialización (Gabel, 2005). En realidad, la inteligencia emocional es el desarrollo de la inteligencia interpersonal e intrapersonal de cada aprendiz, que mediante el conocimiento de sus propias limitaciones es capaz de hacer una mejor planificación de la mejora de su aprendizaje (Torres \& Aguaded, 2018).

En el ámbito de la enseñanza de lenguas extranjeras, no se concibe el aprendizaje de una lengua no materna sin la recreación de un ambiente donde el aprendiz pueda expresar libremente sus emociones, para lo cual es necesario priorizar el aprendizaje cooperativo y exento de presión sobre el individual, memorístico y competitivo (Montero, 2018). De esta manera, se produce un desarrollo del proceso de aprendizaje de la lengua no materna con una evolución acorde a las posibilidades cognitivas del discente (García, 2016). El aprendizaje cooperativo en la enseñanza de lenguas extranjeras permite el desarrollo de las inteligencias intrapersonal, interpersonal y emocional del aprendiz, contribuyendo de esta forma a su formación desde una perspectiva integral, alejándose así del modelo de educación bancaria descrito por Paulo Freire (1970) y la mera transmisión de conocimientos propio de la metodología tradicional (López, 2008; Martínez, 2013). El aprendizaje cooperativo precisa que el discente regule sus emociones para la negociación y el establecimiento de acuerdos que conduzcan a la consecución exitosa de la tarea conjunta (Fonseca, 2006)

Las TIC juegan un papel muy importante en este proceso, ya que actúan como facilitadores de la educación emocional (González \& Villarrubia, 2011). El uso de las nuevas tecnologías en el aula, aplicadas de manera eficiente, contribuye a que el docente coloque al aprendiz en el centro del proceso de aprendizaje, permitiendo una educación individualizada acorde con los ritmos de cada discente (Marulanda et al, 2018). Aplicado al campo de las lenguas extranjeras, el uso de las TIC ha conducido al desarrollo de disciplinas como la Enseñanza de Lenguas Asistida por Ordenador o ELAO (en inglés Computer Assisted Language Learning o sus siglas CALL), que en plena era de los dispositivos móviles ha evolucionado a la Enseñanza de Lenguas Asistida por Tecnología Móvil o ELAM (en inglés Mobile Assisted Language Learning o su acrónimo MALL) (Jordano, 2016; Trujillo, Salvadores \& Gabarrón, 2019). Las diferentes herramientas y aplicaciones de la web 2.0, la web 3.0 y los diferentes escenarios que se divisan en un futuro no muy lejano convierten a las nuevas tecnologías en la herramienta más poderosa para el cambio de modelo en la enseñanza de lenguas extranjeras basado en la gestión de las emociones y en el desarrollo de tareas adecuadas a las diferentes inteligencias del discente (Moreno et al, 2018). El docente de lenguas 
del siglo XXI debe ser capaz de elaborar recursos educativos que permitan al alumno la realización de tareas basadas en las inteligencias múltiples en general y la inteligencia emocional en particular (Torres \& Ramírez, 2018).

\section{El concepto de "profesor emocional"}

Una vez establecida la importancia de las emociones en el aprendizaje en general y en el de una lengua extranjera en particular, es necesario considerar al docente de lenguas extranjeras como un "profesor emocional" (López, 2016), capaz de despertar en el discente sentimientos, comportamientos, actitudes y, en una palabra, emociones positivas, convirtiéndose en un referente emocional (Bisquerra \& Guiu, 2018). De esta manera, el alumno desarrolla una inteligencia emocional que permite a su vez el desarrollo de sus planos intrapersonal e interpersonal, imprescindibles en la enseñanza de lenguas extranjeras, lo cual le conduce a la comunicación eficaz y a la resolución de situaciones problemáticas a través de la lengua no materna objeto de aprendizaje (Heredero \& Garrido, 2016).

El profesor de lenguas, como "profesor emocional", debe ser lo suficientemente competente para proporcionar el andamiaje necesario que favorezca la creación de escenarios de aprendizaje apropiados para el uso de la lengua extranjera por parte de los alumnos (Delmastro, 2008). Al mismo tiempo, debe tener la suficiente habilidad para crear ambientes de aprendizaje donde el alumno sea capaz de desarrollar sus competencias en la lengua extranjera, especialmente la competencia comunicativa, utilizando herramientas y procedimientos contextualizados para implementar una propuesta de evaluación constructiva y positiva para el aprendiz (Romero et al, 2017).

Al mismo tiempo, el "profesor emocional" de lenguas extranjeras es capaz de implicar al alumno en su propio aprendizaje, con el objetivo de que éste desarrolle su propio aprendizaje de forma autónoma e independiente de su profesor (Buitrón \& Navarrete, 2015). El "profesor emocional" es de esta forma un profesor moderador, que se convierte en un actor más del proceso de aprendizaje de su alumno, al que cede todo el protagonismo, para que llegue a sus propias conclusiones a través de un proceso de reflexión (Betancourt \& Zermeño, 2017). Por otra parte, debe ser un gestor de emociones de aprendices provenientes de diferentes comunidades culturales, que facilite la interacción comunicativa creando un clima de aula basado en la apertura, la tolerancia y el respeto (Vez, 2018). De esta forma, se establece en el docente de lenguas extranjeras una conexión entre la Teoría de las Inteligencias Múltiples, la inteligencia emocional y la competencia intercultural (Arboleya, 2016; Molina, 2018).

Por ello es necesario que el docente de lenguas extranjeras reciba una formación socio-afectiva, de tal forma que esté preparado para despertar las emociones del alumno y encauzarlas hacia el proceso de aprendizaje de una lengua no materna (Gallego Espinoza \& Fuentes Blanco, 2018). De esta forma, el profesor se convierte en un referente, en un modelo a seguir desde el punto de vista emocional y afectivo, contribuyendo a la formación socializadora del alumno y a la regulación de sus emociones (Suberviola, 2018). Por otra parte, la gestión de las propias emociones por parte del profesor produce en éste un bienestar personal que al mismo tiempo se ve reflejado en su desempeño profesional, aumentando su autoestima como docente, lo cual le habilita en mayor medida para desarrollar el autoconcepto y la autoestima de sus aprendices en la implementación de un proceso de aprendizaje exitoso (Colomeischi, 2015).

\section{Discusión y conclusiones}

La Teoría de las Inteligencias Múltiples de Gardner fue relevante para la educación en general y para la enseñanza y el aprendizaje de lenguas extranjeras en particular, al determinar que to- das las inteligencias que se encuentran en mayor o menor grado en cada individuo son importantes a la hora de implementar un proceso de aprendizaje de una lengua extranjera con garantías de éxito, alejándonos de los parámetros memorísticos y gramaticales de los métodos de enseñanza tradicionales para pasar a situaciones de aprendizaje en los que prevalece la variable comunicativa y las destrezas orales a partir de la interacción con otros individuos. De la combinación de las inteligencias interpersonal e intrapersonal se nutre la inteligencia emocional. Las emociones del alumno son situadas en el centro del aprendizaje de lenguas extranjeras, al considerar a estas como elemento clave para el aprendizaje cooperativo, donde conceptos como la autoestima, la inhibición o desinhibición del alumno a la hora de expresarse e interactuar con otros aprendices o con el propio profesor son elementos esenciales para el éxito, especialmente de la adquisición de destrezas comunicativas. El docente de lenguas debe tener una formación adecuada para lograr convertirse en un "profesor emocional", en un gestor de sus propias emociones y las de sus aprendices, capaz de introducir el componente afectivo en el aula para situar el proceso de aprendizaje de una lengua extranjera en situaciones comunicativas propias de la vida real.

Diferentes investigadores han establecido una conexión directa entre el uso de las Inteligencias Múltiples en el aula de lenguas extranjeras y el desarrollo de las inteligencias intrapersonal, interpersonal y verbal-lingüística en los aprendices. Mientras que algunos concluyen que, efectivamente, se produce una evolución de las inteligencias anteriormente mencionadas en el aprendiz a partir de la implementación de determinadas estrategias relacionadas con el uso de las Inteligencias Múltiples (Botwina, 2010; Gómez Castro \& Sobremazas Martínez, 2012), otros apuntan en sentido contrario, determinando que el uso de estrategias relacionadas con la Teoría de las Inteligencias Múltiples no conlleva una evolución y desarrollo de las inteligencias intrapersonal, interpersonal y verbal-lingüística, o al menos no en mayor medida que otras estrategias utilizadas con mayor frecuencia en los procesos de enseñanza y aprendizaje de lenguas extranjeras (Torres \& Aguaded, 2018). El Instituto Cervantes elaboró un decálogo con las competencias claves y específicas que debía poseer el profesor de lenguas extranjeras (2012; 11), entre las que se encuentra la "gestión de los sentimientos y emociones en el desempeño de su labor docente", lo cual supone "la gestión de las emociones propias, la motivación docente, el desarrollo de las relaciones interpersonales y la implicación en el desarrollo de la inteligencia emocional del aprendiz" (Iglesias, 2016).

Del docente de lenguas se espera que sea emocionalmente inteligente, capaz de transmitir la enseñanza de la lengua desde la afectividad y la positividad en las interacciones con sus discentes. De esta forma, el aprendiz, desde un entorno emocionalmente afectivo creado por el profesor, será capaz de desarrollar la inteligencia emocional y de evolucionar en su proceso de aprendizaje de la lengua extranjera.

\section{Referencias}

Acevedo, A. (2018). Aprendizaje del español como lengua extranjera a través de la teoría de las inteligencias múltiples y de las TIC. E-eleando: Ele en Red. Serie de monografías y materiales para la enseñanza de ELE, 9, 1-54.

Arboleya, I. (2016). La teoría de las inteligencias múltiples como filosofía para la enseñanza del inglés como segunda lengua. Debates E Prácticas en Educación, 1(1), 4-5.

Betancourt, R. R., \& Zermeño, M. G. G. (2017). Competencias digitales en la enseñanza-aprendizaje del inglés en bachillerato. Campus Virtuales, 6(2), 51-59.

Bisquerra, R., \& Guiu, G. F. (2018). Análisis del vocabulario emocional en el profesorado de lengua. Revista electrónica interuniversitaria de formación del profesorado, 21(1), 161-172. 
Botwina, R. (2010). Towards Creative Foreign Language Teaching: The Theory of Multiple Intelligences in Use. Journal of Pedagogy and Psychology" Signum Temporis", 3(1), 13-18.

Breen, M.P. (1987). Leamer Contributions to Task Design. En C. Candlin y D. Murphy (eds.), Language Learning Tasks (pp. 23.47). Englewood Cliffs, NJ.: Prentice Hall.

Buitrón, S., \& Navarrete, P. (2015). El docente en el desarrollo de la inteligencia emocional: reflexiones y estrategias. Revista $D i$ gital de Investigación en Docencia Universitaria-RIDU, 4(1), 1-8.

Candlin, C. N. (1990). Hacia la enseñanza de lenguas basada en tareas. Comunicación, lenguaje y educación, 2(7-8), 33-53.

Carrillo, M. E., \& López, A. L. (2014). La teoría de las inteligencias múltiples en la enseñanza de las lenguas. Contextos Educativos. Revista de Educación, 17, 79-89.

Chomsky, N. (1986). Knowledge of language. New York: Praeger.

Colomeischi, A. A. (2015). Teacher's burnout in relation with their emotional intelligence and personality traits. Procedia-Social and Behavioral Sciences, 180, 1067-1073.

Delmastro, A. L. (2008). El andamiaje docente en el desarrollo de la lectura y la escritura en lengua extranjera. Paradigma, 29(1), 197-230.

Díaz Posada, L. E., Valera Londoño, S. P., \& Rodríguez Burgos, L. P. (2017). Multiple Intelligences and Curriculum Implementation: Progress, Trends and Opportunities//Inteligencias múltiples e implementación del currículo: Avances, tendencias y oportunidades. Revista de Psicodidáctica, 22(1), 69-83.

Estaire, S. (1990). La programación de unidades didácticas a través de tareas. Cable, 5, 28-39.

Fonseca, M. C. (2006). Las inteligencias múltiples en la enseñanza del español: los estilos cognitivos de aprendizaje. Publicaciones académicas del Instituto Cervantes. Recuperado el 1-07-2019 de http://cvc.cervantes.es/ensenanza/biblioteca_ele/publicaciones_centros/PDF/munich_2006-2007/03_fonseca.pdf

Fontes, O. L., González, L., \& Martínez, R. (2019). El aprendizaje cooperativo en la clase de inglés como lengua extranjera. Transformación, 15(1), 63-73.

Freire, P. (1970). Pedagogy of the oppressed (MB Ramos, Trans.). New York: Continuum, 2007.

Gabel, R. (2005). Inteligencia emocional: perspectivas y aplicaciones ocupacionales. Documentos de trabajo, 16. Universidad ESAN, Lima. Web: http://www.esan.edu.pe/publicaciones/2009/12/07/DocTrab16.pdf

Gallego, V. M. G., \& Fuentes, C. A. P. (2018). Estrategias Para El Aprendizaje De Un Idioma: Lo que todo maestro debe saber. Letras Con* Ciencia Tecno* Lógica, 36-46.

García, A. A. (2018). Aprendizaje del español como lengua extranjera a través de la teoría de las inteligencias múltiples y de las TIC. E-eleando: Ele en Red. Serie de monografías y materiales para la enseñanza de ELE, 9, 1-54.

García, C. M. (2016). Principios del aprendizaje del cerebro y su repercusión en el proceso de adquisición-enseñanza de la lengua extranjera en la educación bilingüe. Encuentro: revista de investigación e innovación en la clase de idiomas, 25, 76-86.

Gardner, H. (1993). Multiple Intelligences: The Theory and Practice. New York: Basic Books.

Gardner, H., \& Hatch, T. (1989). Educational implications of the theory of multiple intelligences. Educational researcher, 18(8), 4-10.

Godoy, I. S., \& Madinabeitia, S. C. (2016). El desarrollo de la autonomía mediante las técnicas de aprendizaje cooperativo en el aula de 12. Porta Linguarum: revista internacional de didáctica de las lenguas extranjeras, 25, 179-190.

Gómez, C., \& Sobremazas, C. (2012). La teoría de las inteligencias múltiples y los estilos de aprendizaje en el aula de inglés: un estudio de caso. Estilos de aprendizaje: investigaciones y experiencias [V Congreso Mundial de Estilos de Aprendizaje], Santander, 27, 28 y 29 de junio de 2012.
Goleman, D. (1996). Emotional intelligence. Why it can matter more than IQ. Learning, 24(6), 49-50.

González, P., \& Villarrubia, M. (2011). La importancia de la variable afectiva en el aprendizaje de L2. Actas de las IV Jornadas Didácticas del Instituto Cervantes de Mánchester. Centro Virtual Cervantes. Recuperado el 1-07-2019 de https://cvc.cervantes. es/ensenanza/biblioteca_ele/publicaciones_centros/PDF/manchester_2011/06_gonzalez_villarrubia.pdf

Griva, E., \& Deligianni, A. (2017). CLIL implementation in foreign language contexts: exploring challenges and perspectives. Research Papers in Language Teaching and Learning, 8(2), 63-73.

Heredero, H., \& Garrido, M. D. P. (2016). Desarrollo de la inteligencia interpersonal e intrapersonal en educación primaria a partir del uso de tecnologías de información y comunicación: estudio de casos. Notandum, 44, 175-188.

Iglesias, I. (2016). El perfil del profesor de español como lengua extranjera: dimensión formativa, dimensión técnica y dimensión afectivo-emocional. Studia Romanica Posnaniensia, 43(3), 67-83.

Instituto Cervantes (2012). Las competencias clave del profesorado de lenguas segundas y extranjeras. Disponible en http:// coc.cervantes.es/ensenanza/biblioteca_ele/competencias/default.htmI

Kiraly, D., \& Hernández, N. G. (2019). SLE: un Enfoque Didáctico para Fomentar la Emergencia de Lenguas Adicionales (Vol. 3). Munich: AVM.

Krashen S. \& Terrell T. (1983). The natural approach: Language acquisition in the classroom. United Kingdom: Pergamon Press.

Krashen, S. (1985). The Input Hypothesis: Issues and Implications. London: Longman.

Lee, L. (2016). Autonomous learning through task-based instruction in fully online language courses. Language Learning $\mathcal{E} T$ echnology, 20(2), 81-97.

Lie, A. (2002). Cooperative learning. Recuperado el 1-07-2019 de https:/www.eduhk.hk/aclass/Theories/cooperativelearningcoursewriting_LBH\%2024June.pdf

Lizasoain, A., \& Mansilla, C. B. (2018). Utilización de una herramienta TIC para la enseñanza del inglés en un contexto rural. Educação e Pesquisa, 44, e167454-e167454.

Long, M. (1985). A Role for Instruction in Second Language Acquisition: Task-based Language Teaching; Modelling and assessing second language acquisition, 77-99.

López, J. O. (2008). Paulo Freire y la pedagogía del oprimido. Revista Historia de la Educación Latinoamericana, 10, 57-72.

López, M. M. (2016). Las emociones en el aprendizaje de una lengua extranjera: su impacto en la motivación. Revista Internacional de Lenguas Extranjeras/International Journal of Foreign Languages, 5, 27-46.

Martínez, M. (2013). La relación entre el aprendizaje cooperativo y la adquisición de competencias interpersonales en una clase de lengua inglesa. Encuentro 22, 73-83.

Marulanda, K. G., Ramírez, Ó. M. M., Londoño, B. M. C., \& López, H. G. (2018). Las TIC como mediación pedagógica y su relación con la autoeficacia en el aprendizaje del inglés como lengua extranjera. Revista de Investigaciones - UCM, 18(32), 60-68.

Mass, L. A., Milián, P. M., López, A. M., Tomé, O. M., Vásquez, Y. \& Herrera, L. R. (2010). Las tareas docentes integradoras dentro de la estructura de la actividad de estudio. Medisur, 8(6), 83-88.

Mayer, J. \& Salovey, P. (1997). What is emotional intelligence? En Salovey, P. \& Sluyter, D. J. (1997): Emotional development and emotional intelligence: Educational implications, 23-54. New York: BasicBooks

Menéndez, O. B. (2007). Las tareas comunicativas en el aprendizaje de lenguas extranjeras: una alternativa para el desarrollo de habilidades comunicativas. Revista Iberoamericana de Educación, 42(5), 1-6. 
Mercadé, A. (2016). Los 8 tipos de inteligencia según Howard Gardner: la teoría de las inteligencias múltiples. Recuperado de: http://biblioteca.udgvirtual.udg.mx/jspui/handle/123456789/2943

Molina, J. E. S. (2018). La interculturalidad en la adquisición del inglés: desde la diversidad hacia la equidad; Interculturalism in the acquisition of English: from diversity towards equality. Revista FAIA, 7(31), 1-26.

Montero, R. L. (2018). Enseñanza de idiomas mediante la acción social. Universidad en Diálogo: Revista de Extensión, 8(1), 11-22.

Morales, H. A., \& Barrientos, Y. Y. (2016). El proceso de enseñanza aprendizaje de la lengua inglesa bajo el enfoque Focal Skills en escuelas públicas. Atenas, 1(33), 73-88.

Moreno, M. Á. (2009). Historia de la metodología de enseñanza de lenguas extranjeras. Tejuelo: Didáctica de la Lengua y la Literatura. Educación, 8, 59-76.

Moreno, A. E., Rodríguez, J. V. R., \& Rodríguez, I. R. (2018). La importancia de la emoción en el aprendizaje: propuestas para mejorar la motivación de los estudiantes. Cuaderno de Pedagogía Universitaria, 15(29), 3-11.

Nunan, D. (1989): Designing Tasks for the Communicative Classroom. Cambridge: C.U.P.

Puren, C. (2012). Del enfoque por tareas a la perspectiva co-accional. Enunciación, 17(2), 155-160.

Ramírez, G. C., \& Acosta, J. R. (2015). La enseñanza de lenguas extranjeras: historia, teoría y práctica. Revista de Lenguas Modernas, 22, 297-316.

Rendine, L. (2018). El enfoque por tareas en la enseñanza de Lenguas extranjeras. Una secuencia didáctica para Bachillerato. Universidad de Valladolid. Facultad de Filosofía y Letras.

Romero Martín, M. R., Castejón Oliva, F. J., López Pastor, V. M., \& Fraile Aranda, A. (2017). Evaluación formativa, competencias comunicativas y TIC en la formación del profesorado. Comunicar, 25(52), 73-83.

Salinas, J. G., Cabrera, A. F., \& Ríos, S. M. (2012). Autonomía en el aprendizaje de lenguas extranjeras en contextos de enseñanza mediatizados por la tecnología. Onomázein: Revista de lingüística, filología y traducción de la Pontificia Universidad Católica de Chile, 25, 15-50.

Salovey, P., \& Mayer, J. D. (1990). Emotional intelligence. Imagination, cognition and personality, 9(3), 185-211.

Schütz, R. (2007). Stephen Krashen's theory of second language acquisition. English made in Brazil, Recuperado el 1-07-2019 de http://www.sk.com.br/sk-krash.html

Slagter, P. J., \& van Ek, J. A. (1979). Un nivel umbral. Estrasburgo. Consejo de Europa.

Slavin, R. E. (1980). Cooperative learning. Review of educational research, 50(2), 315-342.
Suárez, J., Maiz, F., \& Meza, M. (2010). Inteligencias múltiples: Una innovación pedagógica para potenciar el proceso enseñanza aprendizaje. Investigación y Postgrado, 25(1), 81-94.

Suberviola, I. (2018). Autopercepción del profesorado de los Programas de Currículo Adaptado sobre la formación y designación docente de estas aulas. Investigación en la Escuela, 94, 63-78

Terrell, T. D. (1986). Acquisition in the natural approach: The binding/access framework. The Modern Language Journal, 70(3), 213-227.

Torres, P. B., \& Aguaded, E. M. (2018). Aplicación e impacto de las Inteligencias Múltiples en la Enseñanza de Lenguas Extranjeras. Profesorado, 21(1), 95-114.

Trim, J. L. (2007). Modern languages in the Council of Europe 1954-1997. Council of Europe Language Policy Division.. Recuperado el 1-07-2019 de https://www.languagescompany.com/ wp-content/uploads/TRIM_21janv2007_-EN-2.pdf

Trujillo, F. (2002). Aprendizaje Cooperativo para la enseñanza de la lengua. Publicaciones, 32, 147-162.

Trujillo, F., Salvadores, C. \& Gabarrón, Á. (2019). Tecnología para la enseñanza y el aprendizaje de lenguas extranjeras: revisión de la literatura. RIED. Revista Iberoamericana de Educación a Distancia, 22(1), 153-169.

Valdivia, P. J. M., Cárdenas, W. V. C., Hernández, E. F., \& Zubiría, M. M. C. (2019). Inteligencias Múltiples y L2 en Educación Secundaria. CIEX Journ@ l, 8(8), 19-27.

Valladares, C. J. (2016). Aportaciones didácticas en los libros de texto y su relación con la ansiedad ante el aprendizaje de una lengua extranjera: Una exploración cualitativa de tres libros de texto de español como lengua extranjera paso 1 de educación secundaria (Dissertation). Recuperado el 1-07-2019 de http:// urn.kb.se/resolve?urn=urn:nbn:se:du-21109

Van Ek, J. A., \& Alexander, L. G. (1980). Threshold Level English. Oxford. Pergamon.

Vez, J. M. (2018). Didáctica de las lenguas extranjeras: paradigmas de investigación. Linguarum Arena: Revista de Estudos em Didática de Linguas da Universidade do Porto, 1, 81-102.

Vigotsky, L. (1939). Thought and speech. Psychiatry, 2, 29-54.

Yépez, L. E. P., Gordillo, M. J. V., \& Núñez, M. D. P. R. (2017). Aporte del pensamiento lateral al desarrollo de la inteligencia lingüística. Revista Publicando, 4(13), 269-281.

Zanón, J. (1995). La enseñanza de las lenguas extranjeras mediante tareas. Signos. Teoría y práctica de la educación, 14(6), 52-67.

Zanón, J. (2007). Psicolingüística y didáctica de las lenguas: una aproximación histórica y conceptual. MarcoELE. Revista de Didáctica Español Lengua Extranjera, 5, 1-30. 